\title{
PROGNOSTIC IMPACT OF PRETREATMENT FIB-4 INDEX AFTER RADIOFREQUENCY ABLATION FOR HEPATOCELLULAR CARCINOMA
}

\author{
By
}

EFFAT A. MONEM ${ }^{1}$, MOHAMED EL-GHARIB ${ }^{2}$, AHMED SAYED ${ }^{1}$, And ASHRAF M. ALBREEDY ${ }^{*}$

Departments of Tropical Medicine ${ }^{1}$, and Radiodiagnosis ${ }^{2}$, Faculty of Medicine, Ain

Shams University, Cairo 11566, Egypt (*Correspondence: drashrafbreedy@ yahoo.com Mobile: 0020100-3210054)

\section{Abstract}

Intrahepatic distant recurrence, after successful radiofrequency ablation for hepatocellular carcinoma, is related to many factors. Underlying liver fibrosis/cirrhosis severity thought to be one of these contributors. FIB-4 index is a non-invasive marker which reflects the severity of liver fibrosis. The aim of this study was to evaluate the prognostic value of FIB-4 index after radiofrequency ablation for hepatocellular carcinoma with curative intent. This retrospective study included $121 \mathrm{HCV}$ related hepatocellular carcinoma patients with Child class A who underwent radiofrequency ablation between $1 / 2010 \& 12 / 2014$. The study assessed the overall survival and denovo intrahepatic distant recurrence using pretreatment FIB-4 index.

The result showed that pretreatment FIB-4 index of $\leq 3.25$ patients had significant better free intrahepatic distant recurrence and overall survival rates after radiofrequency ablation when compared to those with pretreatment FIB-4 index of $>3.25$, even in subsets of patients with Child score 5 , single lesion or lesions $<3 \mathrm{~cm}$. Also, FIB-4 index was useful prognostic predictor of intrahepatic distant recurrence and overall survival after radiofrequency ablation in Child class A patients in uni and multivraite analysis.

Key words: FIB-4 index, Hepatocellular carcinoma, Intrahepatic distant recurrence, Non- invasive fibrosis markers, Radiofrequency ablation.

\section{Introduction}

Radiofrequency ablation (RFA) proved an effective nonsurgical curative treatment option for hepatocellular carcinoma (Bruix and Sherman, 2011). Intrahepatic recurrence of HCC after RFA is either local recurrence or de novo intrahepatic distant recurrence (IDR). Local recurrence results from direct spread of the original tumour (Poon et al, 2000). Whereas IDR was believed to develop in a preneoplastic liver in a location away from the original tumour through a process called multicenteric occurrence (Nakashima and Kojiro, 2001). The underlying degree of liver fibrosis or cirrhosis might app ear to be significantly associated with de novo recurrence (Imamura et al, 2003). To measure fibrosis or cirrhosis degree before curative treatment modalities in a trial to predict prognosis was indicated (Sumida et al, 2012).

The FIB-4 index is a non-invasive marker of liver fibrosis (Sterling et al, 2006) proved to be correlated with the degree of liver fibro- sis in chronic liver disease patients due to different etiology without HCC, but, still blurred whether it could be a biomarker to predict prognosis in HCC patients perform-ed RFA with curative intent (Xiao et al, 2015).

The study aimed to evaluate the FIB-4 index impact on overall survival (OS) \& denovo IDR after successful RFA for HCV related HCC patients with Child-Pugh class A.

\section{Patients and Methods}

This study protocol and all procedures performed were approved by and in accordance with the ethical standards of the Institutional and National Research Committee, Faculty of Medicine, Ain Shams University, Cairo, and with the ethical standards laid down in an appropriate version of the WMA Declaration of Helsinki (1964) and its later amendments or comparable ethical standard. Informed consent was obtained from all included participants before the RFA procedure. The retrospective study was conducted at Tropical Medicine department \& HCC outpatients' 
clinic, Ain Shams University Hospitals. Data were retrieved from the HCC patients' files performed RFA from January 2010 till December 2014. They were 2695 consecutive adult Egyptian patients, but 121 underwent RFA were selected and enrolled based on inclusion and exclusion criteria.

Inclusion criteria were: 1- Child class A patients, 2- HCC according to AASLD practice guidelines (Bruix and Sherman, 2011), 3- Underwent RFA for $\mathrm{HCC}$ according to Barcelona-clinic-liver-cancer (BCLC) staging system (Bruix and Sherman, 2011), 4Complete tumor response after a month from RFA after modified RESICT criteria i.e. complete disappearance of intra tumoral enhancement in all target lesions using dynamic imaging; CT or MRI (Lencioni and Llovet, 2010), \& 5- positive HCV antibody. Patients excluded were: i- previous HCC treatment; ii- local recurrence after RFA; defined direct dissemination of original tumor along the ablated lesion peripheral margin (Goldberg et al, 2009), iii- conditions affect- ed platelet count as splenectomised patients, acute or chronic renal failure, chronic obstructive lung disease, hematologic disorders \& malignancies, iv- on drugs as inhibitors of platelet function or aspirin, ticlopidine, clopidogrel, non-steroid antiinflammatory within 6 months prior RFA, \& v- liver disease other than HCV as HBV.

The pre-treatment demographic, clinical and laboratory were collected from the records of the patients which included: Full personal history taking and clinical examination; complete blood picture; complete liver profile; etiology of underlying liver disease (viral, autoimmune, etc.); renal function tests; abdominal ultrasound; alpha fetoprotein (AFP) level, triphasic spiral abdominal CT and/or MRI and calculation of FIB4 index at time of diagnosis.

Calculation of FIB-4 index: FIB-4 index was calculated from the patient data at the time of diagnosis which was within 2 weeks of RFA using the following formula (Wai et al, 2003):

\section{FIB-4= Age (years) x AST (IU/L)}

A FIB-4 index $>3.25$ with positive predictive value confirmed significant fibrosis (F3F4) of $82 \%$, a specificity of $98 \%$ (ValletPichard et al, 2007). This cutoff value evaluated impact of underlying fibrosis/cirrhosis on OS and IDR free survival after RFA.

Follow up of patients after RFA: All patients were followed up by AFP and triphasic spiral CT and/or MRI performed 1 month after RFA then every 3 months for the next 2 years then every 6 months thereafter to evaluate the recurrence. All enrolled patients were evaluated for IDR free survival and OS after RFA and their relation to pre-treatment FIB 4 index. IDR was defined as intrahepatic recurrence beyond the ablative margin of $0.5-1.0 \mathrm{~cm}$ away from ablated lesion (Goldberg et al, 2009).

Radiofrequency ablation technique: This procedure was carried-out in the interventional radiology unit, Ain-Shams University Hospital. Tumor must be visualized with US and accessible via tpercutaneous route. The patients were placed in supine position and had anesthesia in the form of propofol $1 \%$ infusion with $10 \mathrm{~cm}$ infiltration local anesthesia at the site of electrode entry to decrease post procedure pain. The radiofrequency device used was cool tip RF system, valley lab USA, Mass-with maximum power $480 \mathrm{kHz}$ waves, with display parameters including: impedance, current, power and temperature. RF electrodes used was cooledtip electrode needle of 2 types, single for ablating lesion $\leq 3 \mathrm{~cm}$ and cluster for ablating lesions $3-5 \mathrm{~cm}$ in diameter. Subcostal approach was generally used for left lobe lesions. However, for right lobe lesions either subcostal or intercostal approaches were used.

Statistical analysis: Data were computerized and analysed using Statistical Package for Social Sciences (SPSS) software computer program version 20. Data were expressed as output capability of $200 \mathrm{~W}$ and produces a 
$\mathrm{M} \pm \mathrm{SD}$ for quantitative parametric measures in addition to Median Percentiles for quantitative non-parametric measures and both number and percentage for categorized data. Student $t$ test compared means between 2 groups for parametric data. Mann Whitney Test (U test) compared between two independent groups for non-parametric data. Chi square test was used to examine the relationship between two qualitative variables. Survival analysis estimated the OS and IDR-free survival probabilities after RFA, using KaplanMeier method. Log-rank test determined parameters significance regarding differences in cumulative OS \& IDR-free survival rates. Logistic regression assessed significance. They sorted sensitivity to discriminate according to $\mathrm{P}$ values, as absolute figures and as a standard error of estimate (95P). A P $=0.05$ was considered significant.

\section{Results}

This study included 121 patients, 89 (73.6\%) were males and $32(26.4 \%)$ were females and the mean age of all enrolled patients was 57.23 years. Child A5 patients were 49 (40.5\%) and $72(59.5 \%)$ patients were Child score 6. The Mean FIB4 score was 5.1. The mean MELD score was 9.59. The AFP median was 20.8. Patients with single lesion were $92(76 \%)$, with 2 lesions $27(22.3 \%) \&$ 2 lesions $(1.7 \%)$, with largest lesion mean size was $2.78 \mathrm{~cm}$.

Patients were categorized into GI 34 with FIB-4 index of $\leq 3.25$ and G2 87 with $>3.25$ G1 had significant lower mean serum albumin values $(3.5 \pm 0.47 \mathrm{~g} / \mathrm{ml})$ and platelets $(100287.4 \pm 28023.21$ cell $/ \mathrm{m} 3)$ than G2 (3.72 $\pm 0.48 \& 170147.1 \pm 65304.94$ respectively). Also, 25 patients $(73.5 \%)$ had Child score 5 \& $9(26.5 \%)$ had Child score 6 in G1. In G2, 63 patients $(72.4 \%)$ had Child score $6 \& 24$ (27.6\%) had Child score 5 (Tab. 1).

OS rates at $1,2 \& 3$ years after RFA were $100 \%, 93.5 \% \& 80.5 \%$ respectively for patients with pretreatment G1 were significant better $(\mathrm{P}<0.05)$ than those with pretreatment G2 $(95.4 \%, 80.0 \% \& 61.3 \%$ respectively). IDR free survival rates at $1,2 \& 3$ years af- ter RFA were $97.1 \%, 76.5 \%$ \& $39.7 \%$ respectively in patients with pretreatment G1 were significant better $(\mathrm{P}=0.001)$ than those with pretreatment $\mathrm{G} 2(73.6 \%, 35.4 \%$ \& $13.5 \%$ respectively) (Fig. 1).

In 49patients with Child score 5, OS rates at $1,2 \& 3$ years after RFA were $100 \%$, $96.0 \%$ \& $96.0 \%$ respectively for patients G1 and $100 \%, 83.3 \% \& 68.9 \%$ respectively for G2. Also, denovo IDR free survival rates at 1,2 \& 3 years were $96.0 \%, 80.0 \%$ \& 39.4\% respectively for patients G1 and $91.7 \%$, $40.7 \%$ \& $15.3 \%$ respectively for G2. Curves compared by Log rank test showed that patients G1 showed significant better OS and IDR free survival after RFA than G2 (Fig. 2a). In 72patients with Child score 6, OS rates at $1,2 \& 3$ years after RFA were $100 \%, 85.7 \%$ \& $85.7 \%$ respectively for patients G1 and $93.7 \%, 78.5 \%$ \& $58.2 \%$ respectively for G2. IDR free survival rates at $1,2 \& 3$ years were $100 \%, 66.7 \% \& 40.05 \%$ respectively for patients G1 and $66.7 \%$, $33.3 \%$ \& $12.7 \%$ respectively for G2. Curves comparison did not show significant difference between among groups regarding OS rates $(\mathrm{P}=0.192)$. Patients $\mathrm{G} 1$ showed better significant IDR free survival rates after RFA than $\mathrm{G} 2$ with Child score $6(\mathrm{P}=0.018)$ (Fig. $2 b)$.

In 89patients with lesions $<3 \mathrm{~cm}$, overall survival rates at $1,2 \& 3$ years after RFA were $100 \%, 93 \% \& 93 \%$ respectively for patients $\mathrm{G} 1$ and $98.2 \%, 87.3 \%$ \& $70 \%$ respectively for G2. IDR free survival rates were $96.9 \%, 78.1 \%$ \& $42.6 \%$ respectively for patients $\mathrm{G} 1$ and $82.5 \%, 47 \%$ \& $17.5 \%$ respectively for G2. No significant difference was in groups regarding overall survival rates after RFA ( $\mathrm{P}=0.066)$, patients $\mathrm{G} 1$ showed significant better denovo IDR free survival rates after RFA than G2 (Fig. 3).

In 91patients with single lesion, OS rates at $1,2 \& 3$ years after RFA were $100 \%, 96 \%$ \& $96 \%$ respectively for patients $\mathrm{G} 1$ and $95.5 \%, 81.4 \%$ \& $61 \%$ respectively for $\mathrm{G} 2$. IDR free survival rates at $1,2 \& 3$ years were $96 \%, 80 \% \& 47.1 \%$ respectively for 
patients G1 and $80.3 \%, 44.2 \%$ \& $14.6 \%$ respectively for G2. Log rank test showed that patients G1 showed significant better overall survival $(p=0.008)$ and denovo IDR free survival $(\mathrm{P}<0.001)$ after RFA than G2 (Fig. 4)

Tumor size, Child score, and tumor number of pretreatment Fib 4 index $(p<0.05)$ were significantly associated with OS after RFA by univariate logistic regression. Multivariate logistic regression showed only larger tumor size $(\mathrm{p}=0.005, \mathrm{OR}=3.65)$, higher tumor number $(\mathrm{p}=0.007, \mathrm{OR}=2.968)$ and higher pretreatment FIB-4 index $(\mathrm{p}=0.019$, $\mathrm{OR}=3.045$ ) were independent risk factors associated with lower OS rates (Tab. 2).

Tumor No. $(p=0.040, O R=4.136) \&$ pretreatment FIB-4 index $(\mathrm{p}=0.047, \mathrm{OR}=2.772)$ were factors significantly associated with IDR after RFA using univariate logistic regression analysis. By using multivariate logistic regression analysis, only high pretreatment FIB 4 index $(\mathrm{p}=0.035$, $\mathrm{OR}=6.267)$ was an independent risk factor associated with higher IDR rates after RFA (Tab. 3).

Table 1: Patients with FIB-4 index of $\leq 3.25 \&>3.25$ regarding laboratory data, MELD \& Child scores

\begin{tabular}{|c|c|c|c|c|}
\hline \multicolumn{2}{|l|}{ Variants } & Fib4 $\leq 3.25(n=34)$ & Fib4 $>3.25(n=87)$ & P-value \\
\hline $\mathrm{AST}^{*}(\mathrm{IU} / \mathrm{ml})$ & $\mathrm{M} \pm \mathrm{SD}$ & $49.47 \pm 30.56$ & $56.54 \pm 34.58$ & 0.331 \\
\hline $\mathrm{ALT}^{* *}(\mathrm{IU} / \mathrm{ml})$ & Median (IQR) & $43(31-66)$ & $50(28-71)$ & 0.621 \\
\hline Bilirubin*(mg/dl) & $\mathrm{M} \pm \mathrm{SD}$ & $1.01 \pm 0.35$ & $1.15 \pm 0.46$ & 0.119 \\
\hline Albumin* $(\mathrm{g} / \mathrm{dl})$ & $\mathrm{M} \pm \mathrm{SD}$ & $3.72 \pm 0.48$ & $3.4 \pm 0.47$ & 0.001 \\
\hline Platelet* (cells $\left./ \mathrm{m}^{3}\right)$ & $\mathrm{M} \pm \mathrm{SD}$ & $170147.1 \pm 65304.94$ & $100287.4 \pm 28023.21$ & 0.000 \\
\hline Creatinin* mg/dl) & $\mathrm{M} \pm \mathrm{SD}$ & $0.94 \pm 0.17$ & $0.97 \pm 0.14$ & 0.238 \\
\hline $\mathrm{AFP}^{* *}(\mathrm{ng} / \mathrm{ml})$ & Median (IQR) & $21.25(7.4-69)$ & $20.8(10-93)$ & 0.804 \\
\hline INR* & $\mathrm{M} \pm \mathrm{SD}$ & $1.26 \pm 0.14$ & $1.29 \pm 0.14$ & 0.323 \\
\hline Meld* & $\mathrm{M} \pm \mathrm{SD}$ & $9.09 \pm 2.05$ & $9.78 \pm 2.45$ & 0.147 \\
\hline \multirow{2}{*}{ Child score*** } & A5 & $25(73.5 \%)$ & $24(27.6 \%)$ & \multirow{2}{*}{0.000} \\
\hline & A6 & $9(26.5 \%)$ & $63(72.4 \%)$ & \\
\hline
\end{tabular}

Table 2: Univariate and multivariate logistic regression analysis for predictors of overall survival

\begin{tabular}{|l|c|c|c|c|}
\hline \multirow{2}{*}{ Variants } & \multicolumn{2}{|c|}{ Univariate analysis } & \multicolumn{2}{c|}{ Multivariate analysis } \\
\cline { 2 - 5 } & P-value & Odds ratio (95\% CI) & P-value & Odds ratio (95\% CI) \\
\hline Age & 0.087 & $1.050(0.993-1.110)$ & & \\
\hline Sex & 0.762 & $0.876(0.372-2.062)$ & & \\
\hline Child score & 0.024 & $2.630(1.136-6.088)$ & & \\
\hline Tumor size & 0.000 & $5.042(2.127-11.956)$ & 0.005 & $3.650(1.473-9.043)$ \\
\hline Number of tumors & 0.007 & $4.031(1.858-8.808)$ & 0.007 & $2.968(1.067-6.683)$ \\
\hline Pretreatment AFP & 0.467 & $1.231(0.704-2.152)$ & & \\
\hline Pretreatment Fib 4 & 0.005 & $4.876(1.634-15.594)$ & 0.019 & $3.045(1.256-7.673)$ \\
\hline
\end{tabular}

AFP: Alpha feto-protien

Table 3: Univariate and multivariate logistic regression analysis for predictors of IDR

\begin{tabular}{|l|c|c|c|c|}
\hline & \multicolumn{2}{|c|}{ Univariate analysis } & \multicolumn{2}{c|}{ Multivariate analysis } \\
\hline & P-value & Odds ratio (95\% CI) & P-value & Odds ratio (95\% CI) \\
\hline Age & 0.429 & $1.029(0.959-1.104)$ & & \\
\hline Sex & 0.563 & $0.705(0.215-2.306)$ & & \\
\hline Child score & 0.245 & $1.795(0.670-4.807)$ & & \\
\hline Tumor size & 0.569 & $1.038(0.941-1.105)$ & & \\
\hline N of tumors & 0.040 & $4.136(1.343-33.770)$ & & \\
\hline Pretreatment AFP & 0.052 & $2.371(0.992-5.668)$ & & \\
\hline Pretreatment Fib 4 & 0.047 & $2.772(1.012-7.590)$ & 0.035 & $6.267(1.775-50.699)$ \\
\hline
\end{tabular}

Discussion

Intrahepatic recurrence after RFA represented a major drawback affecting patient survival results (Yoshida et al, 1999). Others reported that incidence of HCC development increases with progression of underlying fibrosis/ cirrhosis, and confirmed liver fibrosis as a risk factor for HCC recurrence after curat-ive hepatectomy (Ko et al, 2002; Gas- smann et al, 2010; Kaibori et al, 2013; Toyoda et al, 2015).

To the authors knowledge none evaluated FIB-4 index as a predictor of OS and IDR after RFA in HCC patients with Child A, HCV-related cirrhosis. There was a great difference between Child A and B patients and even a great heterogeneity exits between Child B patients themselves regarding liver 
dysfunction, patient performance status of the and the overall survival (Cholongitas and Burroughs, 2012). So, only Child class A patients were selected to prevent the confounding influence of these factors on survival rates.

In the current study, the pretreatment FIB4 index patients were categorized into (G1) with FIB-4 $\leq 3.25$ and (G2) with FIB-4 > 3.25. The HCC patients G1 showed significant better OS and IDR free survival rates at 1,2 \& 3 years after RFA when compared to those of G2. The univariate analysis showed that tumor size, Child score, tumor number and pretreatment G1 were significantly associated with poor OS rates after RFA, but only tumor size, tumor number and pretreatment G2 were independent risk factors associated with lower OS rates on multivariate analysis. As to the IDR, univariate analysis showed that tumor number and pretreatment G1 were significant predictors of IDR after RFA, while only pretreatment G2 showed an independent risk factor associated with IDR after RFA. This agreed with Ito et al. (2015) who examined Child A HCC patients underwent hepatect- omy/local ablative techniques, and found that patients with FIB-4 index $<2.0$ had a hi-ghest survival rate, followed by those with FIB-4 index $\geq 2.0 \&$ $<4.0$, and then those with FIB-4 index $\geq 4.0$, with recurrence rate of FIB-4 index $\geq 4.0$ was higher than others.

Also, Toyoda et al. (2015) and Liao et al. (2017) found that patients with a preoperative FIB- 4 index of $\leq 3.25$ had significant better OS and recurrence free survival rates after curative resection than those with a pretreatment FIB-4 index of $\leq 3.25$.

In agreement with the present study, higher FIB-4 index was associated with lower survival rate after curative hepatectectomy in univariate analysis as well as in multivariate analysis along with age, tumor size $>5$ $\mathrm{cm}$, multiple tumor, portal vein invasion, blood loss and transfusion during hepatectomy. It was associated with recurrence after curative hepatectectomy in univariate analy- sis as well as in multivariate analysis along with tumor size and multiple, portal vein invasion, blood loss and blood transfusion during hepatectomy (Toyoda et al, 2015). The FIB-4 index was an independent predictive factor for HCC prognosis in Child A class patients after surgery/ablative techniques (Ito et al, 2015). FIB-4 > 3.25 also was an independent risk factor for late recurrence ( $>24$ months) after surgery in uni- and multivariate analysis (Liao et al, 2017).

The present results disagreed with Soe et al. (2013) who correlated between different noninvasive fibrosis indices (including FIB 4 index) and intrahepatic distant recurrence of HCC after RFA. They found that only high levels of AFP and age platelet index at baseline predict IDR of HBV-related HCC after RFA, but other non-invasive markers included FIB-4 were not significant. Also, high aspartate aminotransferase-to-platelet ratio (APRI) index was only an independent risk factor for IDR after RFA, but FIB-4 and other markers were not significant (Chung et $a l, 2016)$. This discrepancy might be due to that their patients were infected with $\mathrm{HBV}$ and with Child class A and B.

In the current study, there were 49 patients with Child score 5, with pretreatment FIB-4 $\leq 3.25$ showed significant better OS and IDR free survival rates after RFA than those with FIB-4 >3.25. But, in patients with Child score 6 , statistical difference was found regarding IDR free survival but did not elucidate difference as to OS rates. This agreed with Ito et al. (2015) whose patients with pretreatment FIB-4 index of $\leq 3.25$ showed significant better IDR free survival rates after RFA than with FIB-4 $>3.25$ with Child score $5 \& 6$. This proved that Child class A patients have diverse degrees of liver fibrosis even with minimum score of Child 5 .

In the present study the influence of tumor factors impact of the FIB-4 score was evaluated in patients with lesions $<3 \mathrm{~cm}$ and in patients with single lesion. Patients with pretreatment FIB-4 $\leq 3.25$ showed significant better IDR free survival rates after RFA than 
those with FIB-4 $>3.25$ in both groups. Also, patients with pretreatment FIB-4 $\leq 3.25$ had significant better OS rates after RFA than those with FIB-4 $>3.25$ patients with single lesion. FIB-4 index proved a powerful predictor value, especially in IDR, even in patients with small or single lesion.

\section{Conclusion}

FIB-4 index proved a useful prognostic marker in HCV related Child A patients undergone RFA for HCC. Patients higher FIB4 index were associated with mortality and recurrence after RFA and follow up after RFA was indicated. FIB-4 index might be incorporated in evaluation of those patients outcome. The Egyptian HCC patients more than $90 \%$ were attributable to chronic $\mathrm{HCV}$. Prognostic FIB-4 must investigate HCC patients to other etiology cause than HCV. Also, role of sustained virological response (SVR) to antiviral treatment as more than $80 \%$ of cohort was not eligible to interferon. More studies is a must to highlight the impact of SVR in era of direct acting antiviral therapies on HCC patients, prognosis.

\section{References}

Bruix, J, Sherman, M, 2011: Management of hepatocellular carcinoma: an update. Hepatology 53, 3:1020-2.

Cholongitas, E and Burroughs, AK, 2012: The evolution in the prioritization for liver transplantation. Ann Gastroenterol. 25(1):6-13 Chung, HA, Kim, JH, Hwang, Y, Choi, HS, Ko, SY, et al, 2016: Noninvasive fibrosis marker can predict recurrence of hepatocellular carcinoma after radiofrequency ablation. Saudi J. Gastroenterol. 22, 1:57-63

Gassmann, P, Spieker, T, Haier, J, Schmidt, F, Mardin, WA, et al, 2010: Prognostic impact of underlying liver fibrosis and cirrhosis after curative resection of hepatocellular carcinoma. World J. Surg. 34:2442-51.

Goldberg, SN, Grassi, CJ, Cardella, JF, Charboneau, JW, Dodd, GD3rd, et al, 2009: Image-guided tumor ablation: standardization of terminology and reporting criteria. J. Vasc. Interv. Radiol. 20, 7:S377-90.

Imamura, H, Matsuyama, Y, Tanaka, E, Ohkubo, T, Hasegawa, K, et al, 2003: Risk factors contributing to early and late phase intrahepatic recurrence of hepatocellular carcinoma after hepatectomy. J. Hepatol. 38, 2:200-7

Ito, T, Kumada, T, Toyoda, H, Tada, T, 2015: FIB-4 index for assessing the prognosis of hepato-cellular carcinoma in patients with Child-Pugh class A liver function. J. Cancer Res. Clin. Oncol. 141, 7:1311-9.

Kaibori, M, Kubo, S, Nagano, H, Hayashi, M, Haji, et al, 2013: Clinicopathological features of recurrence in patients after 10-year disease-free survival following curative hepatic resection of hepatocellular carcinoma. World J. Surg. 37:820-8.

Ko, S, Kanehiro, H, Hisanaga, M, Nagao, M, Ikeda, N, et al, 2002: Liver fibrosis increases the risk of intrahepatic recurrence after hepatectomy for hepatocellular carcinoma. Br. J. Surg. 89:57-62.

Lencioni, $\mathbf{R}$ and Llovet, JM, 2010: Modified RECIST (mRECIST) assessment for hepatocellular carcinoma. Semin. Liver Dis. 30, 1:52-60.

Liao, R, Fu, YP, Wang, T, Deng, ZG, Li, D W, et al, 2017: Metavir and FIB-4 scores are associated with patient prognosis after curative hepatectomy in hepatitis B virus-related hepatocellular carcinoma: A retrospective cohort study at two centers in China. Oncotarget. 8, 1:1774-87. Nakashima, O, Kojiro, M, 2001: Recurrence of hepatocellular carcinoma: multicentric occurrednce or intrahepatic metastasis? A viewpoint in terms of pathology. J. Hepatobil. Pancreat. Surg. 8, 5:404-9.

Poon, RT, Fan, ST, Ng, IO, Lo, CM, Liu, CL, et al, 2000: Different risk factors and prognosis for early and late intrahepatic recurrence after resection of hepatocellular carcinoma. Cancer 89:500-17

Seo, JY, Kim, W, Kwon, JH, Jin, EH, Yu, S J, et al, 2013: Noninvasive fibrosis indices predict intrahepatic distant recurrence of hepatitis B related hepatocellular carcinoma following radiofrequency ablation. Liver Int. 33, 6:884-93.

Sterling, RK, Lissen, E, Clumeck, N, Sola, R, Correa, MC, et al, 2006: Development of a simple noninvasive index to predict significant fibrosis in patients with $\mathrm{HIV} / \mathrm{HCV}$ coinfection. Hepatology 43: 1317-25

Sumida, Y, Yoneda, M, Hyogo, H, Itoh, Y, Ono, M, et al, 2012: Validation of the FIB4 index in a Japanese nonalcoholic fatty liver disease population. BMC Gastroenterol. 12:2-6

Toyoda, H, Kumada, T, Tada, T, Kaneoka, Y, 
Maeda, A, 2015: A laboratory marker, FIB4 index, as a predictor for long-term outcomes of hepatocellular carcinoma patients after curative hepatic resection. Surgery 157, 4:699-707

Vallet-Pichard, A, Mallet, V, Nalpas, B, Verka rre, V, Nalpas, A, et al, 2007: FIB-4: an inexpensive and accurate marker of fibrosis in $\mathrm{HCV}$ infection: Comparison with liver biopsy and Fibro-Test. Hepatology 46:32-6.

Wai, CT, Greenson, JK, Fontana, RJ, Kalbfleisch, JD, Marrero, JA, et al, 2003: A simple noninvasive index can predict both significant fibrosis and cirrhosis in patients with chronic he- patitis C. Hepatology 38: 518-26.

Xiao, G, Yang, J, Yan, L, 2015: Comparisons of diagnostic accuracy of APRI \& FIB-4 for detecting liver fibrosis in adult patients with chronic hepatitis B virus infection: A systematic review and meta-analysis. Hepatology 61, 1:292302.

Yoshida, H, Shiratori, Y, Moriyama, M, Arakawa, Y, Ide, T, et al, 1999: Interferon therapy reduces the risk for hepatocellular carcinoma: national surveillance program of cirrhotic and noncirrhotic patients with chronic hepatitis $\mathrm{C}$ in Japan. Ann. Intern. Med. 131:174-81

\section{Explanation of figures}

Fig 1: Kaplan-Meier curves comparing patients with pretreatment FIB-4 index of $\leq 3.25$ and $>3.25$ regarding overall survival and denovo IDR free survival rates after RFA using log rank test .

Fig 2: Comparison between patients with a pretreatment FIB-4 index of $\leq 3.25$ and $>3.25$ regarding OS and IDR free survival rates after RFA in a subset of patients with Child score A5 (fig 2a) and Child score A6 (fig 2b) using kaplan curve and log rank test.

Fig.3: Comparison between patients with a pretreatment FIB-4 index of $\leq 3.25$ and $>3.25$ regarding overall survival and IDR free survival rates after RFA in a subset of patients with lesion $<3 \mathrm{~cm}$ using kaplan curve and log rank test.

Fig. 4: Comparison between patients with a pretreatment FIB-4 index of $\leq 3.25$ and $>3.25$ regarding OS and denovo IDR free survival rates after RFA in a subset of patients with single lesion using kaplan curve and log rank test
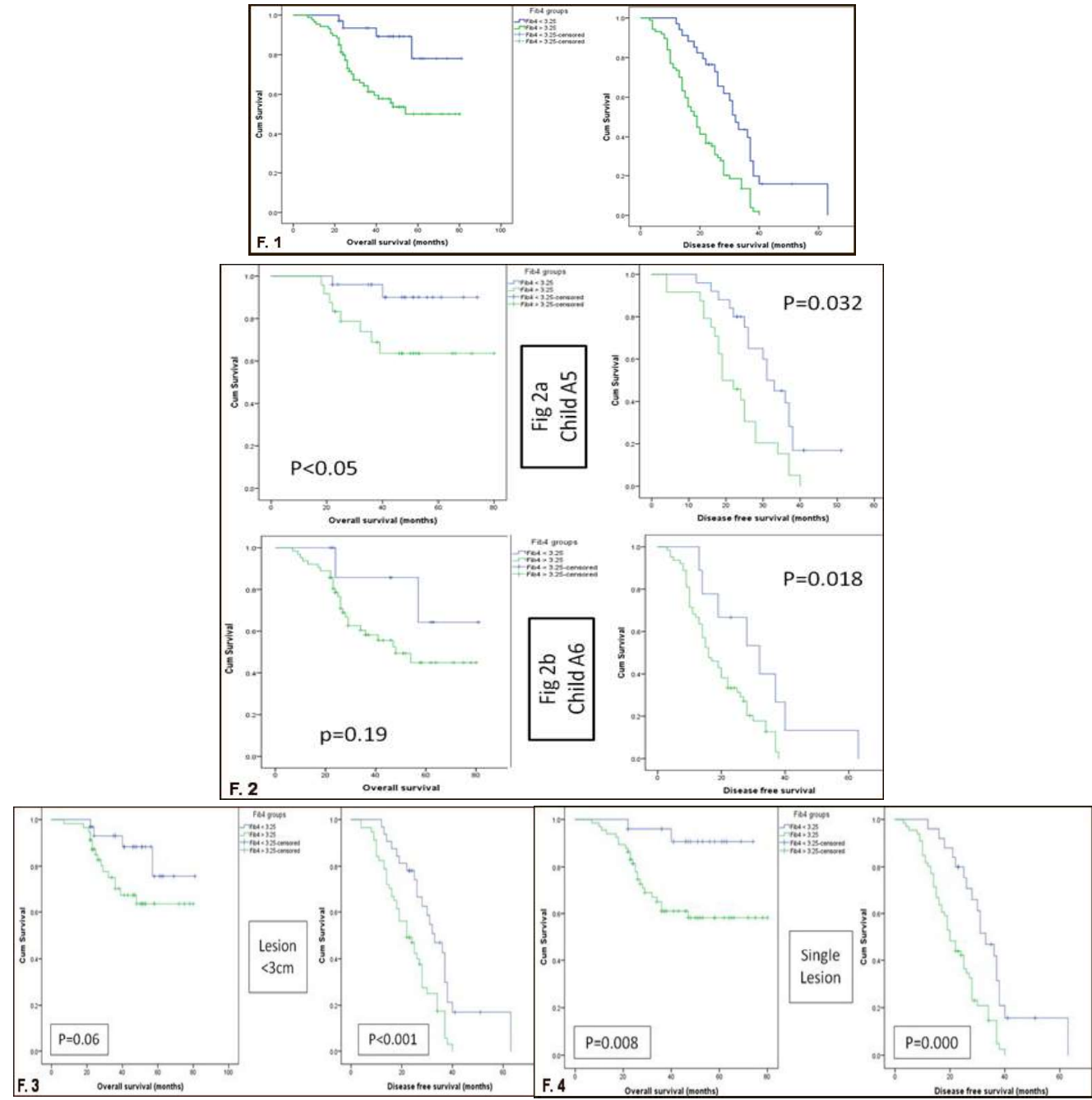